\title{
SUM OF TWO INNER FUNCTIONS AND EXPOSED POINTS IN $H^{1}$
}

\author{
by TAKAHIKO NAKAZI*
}

(Received 4th April 1990)

\begin{abstract}
If $h$ is an outer function in $H^{1}$ then it is shown that $h=\left(q_{1}+q_{2}\right) g$ where both $q_{1}$ and $q_{2}$ are inner functions with $\operatorname{Im} \bar{q}_{1} q_{2} \leqq 0$ almost everywhere, and $g$ is a strong outer function (equivalently, $g /\|g\|_{1}$ is an exposed point of the unit ball of $H^{1}$ ). If $q_{1}+q_{2}$ is nonconstant then such an $h$ is not strongly outer. Moreover a sum of two inner functions is studied.
\end{abstract}

1980 Mathematics subject classification (1985 Revision). Primary 30D55, 46J45; Secondary 47B35.

\section{Introduction}

Let $U$ be the open unit disc in the complex plane and let $\partial U$ be the boundary of $U$. A function in $U$ is said to be of class $N$ if the integrals

$$
\int_{-\pi}^{\pi} \log ^{+}\left|f\left(r e^{i \theta}\right)\right| d \theta
$$

are bounded for $r<1$. If $f$ is in $N$, then $f\left(e^{i \theta}\right)$ which we define to be $\lim _{r \rightarrow 1} f\left(r e^{i \theta}\right)$, exists almost everywhere on $\partial U$. If

$$
\lim _{r \rightarrow 1} \int_{-\pi}^{\pi} \log ^{+}\left|f\left(r e^{i \theta}\right)\right| d \theta=\int_{-\pi}^{\pi} \log ^{+}\left|f\left(e^{i \theta}\right)\right| d \theta
$$

then $f$ is said to be the class $N_{+}$. The set of all boundary functions in $N$ or $N_{+}$is denoted by $N$ or $N_{+}$, respectively. For $0<p \leqq \infty$, the Hardy space $H^{p}$, is defined by $N+\cap L^{p}$.

We call $q$ in $N_{+}$an inner function if $\left|q\left(e^{i \theta}\right)\right|=1$ a.e. on $\partial U$. A function $h$ in $N_{+}$is called outer if it is not divisible in $N_{+}$by a nonconstant inner function. A function $g$ in $H^{1}$ is strongly outer if the only functions $f$ in $H^{1}$ such that $f / g$ is positive are scalar multiples of $g$. (If $g$ has norm 1 , it has this property if and only if it is an exposed point of the unit ball of $H^{1}$.) Every strongly outer function is outer.

H. Helson [7] recently gave a necessary and sufficient condition for strong outer

This research was partially supported by Grant-in-Aid for Scientific Research, Ministry of Education. 
functions. That is related to the divisibility by the square of the sum of two inner functions. We can prove similarly the following result. Let $g$ be outer in $H^{1}$. Then $g$ is strongly outer in $H^{1}$ if and only if $g /\left(q_{1}+q_{2}\right)$ is not in $H^{1}$ for any inner functions $q_{1}, q_{2}$ such that $q_{1}+q_{2}$ is not constant and $-i\left(q_{1}-q_{2}\right) /\left(q_{1}+q_{2}\right)$ is nonnegative almost everywhere. A. Beurling [1] established a celebrated factorization theorem. That is, a nonzero function $f$ in $N_{+}$can be factorized as in the following: $f=q h$ where $q$ is inner and $h$ is outer in $N_{+}$. Thus it is desirable to show that if $f$ is not a strong outer function in $H^{1}$ then $f=\left(q_{1}+q_{2}\right) g$ where both $q_{1}$ and $q_{2}$ are inner functions, $-i\left(q_{1}-q_{2}\right) /\left(q_{1}+q_{2}\right)$ is nonnegative almost everywhere and $g$ is a strong outer function in $H^{1}$. In Section 2 we show this factorization is true. In the proof, a theorem of $\mathrm{E}$. Hayashi $([4,5])$ and theorems of $\mathrm{H}$. Helson $([6,7])$ are crucial. It is more desirable to show that if $f$ is not strongly outer then $f=\left(q_{1}+q_{2}\right)^{2} g$ where both $q_{1}$ and $q_{2}$ are inner and $g$ is strongly outer by a theorem of Helson [7]. In Section 3 we consider this problem. A strong outer function is important in a solution set of an extremal problem of $H^{1}$. In Section 4 we try to describe the solution sets intelligibly. In Section 5 a characterization of absolute values of strong outer functions is given.

\section{A sum of two inner functions}

Suppose $f$ is a function in $H^{1}$ which has the form: $f=\left(q_{1}+q_{2}\right) g$ where $q_{1}, q_{2}$ are inner functions, $q_{1}+q_{2}$ is not constant, $\operatorname{Im} \bar{q}_{1} q_{2} \leqq 0$ and $g$ is a strong outer function. By the following lemma, $f$ is not strongly outer. In this section we show the converse.

Lemma. Let $q_{1}, q_{2}$ be inner functions with $q_{1} \neq-q_{2}$. Then $-i\left(q_{1}-q_{2}\right) /\left(q_{1}+q_{2}\right)$ is nonnegative if and only if $\operatorname{Im} \bar{q}_{1} q_{2} \leqq 0$.

Proof. Use the following equality:

$$
\frac{-i\left(q_{1}-q_{2}\right)}{q_{1}+q_{2}}=\frac{-2 \operatorname{Im} \bar{q}_{1} q_{2}}{\left|1+\bar{q}_{1} q_{2}\right|^{2}}
$$

Theorem 1. If $f$ is not a strong outer function in $H^{1}$ then $f=\left(q_{1}+q_{2}\right) g$ where both $q_{1}$ and $q_{2}$ are inner functions, $\operatorname{Im} \bar{q}_{1} q_{2} \leqq 0$ almost everywhere, $\left(q_{1}-q_{2}\right)^{-1}$ is summable and $g$ is a strong outer function. If $f$ is outer then $q_{1}+q_{2}$ is also outer. If $q_{1}$ is a finite Blaschke product of degreee $n$ then so is $q_{2}$.

Proof. Suppose $f=q_{0} h^{2}$ where $q_{0}$ is inner and $h$ is outer in $H^{2}$. By a theorem of E. Hayashi $([4,5])$

$$
H^{2} \cap(h / \bar{h}) \bar{H}^{2}=g_{0}\left(H^{2} \ominus z q H^{2}\right)
$$

and

$$
\bar{h} / h=\bar{q} \bar{g}_{0} / g_{0}
$$


where $q$ is an inner function and $g_{0}^{2}$ is strongly outer. Hence $h=s g_{0}$ where $s \in H^{2} \ominus z q H^{2}$ and $\bar{q} s^{2} \geqq 0$. Since $\left|s^{2} \pm i q\right|^{2}=|s|^{4}+1$,

$$
s^{2}+i q=p_{1} h_{1} \quad \text { and } \quad s^{2}-i q=p_{2} h_{1}
$$

where $p_{1}, p_{2}$ are inner functions and $h_{1}$ is a function in $H^{1}$ with $h_{1}^{-1} \in H^{\infty}$. Hence

$$
s^{2}=\left(p_{1}+p_{2}\right) \frac{h_{1}}{2} \text { and } q=-i\left(p_{1}-p_{2}\right) \frac{h_{1}}{2}
$$

and

$$
\bar{q} s^{2}=\left(p_{1}+p_{2}\right) /-i\left(p_{1}-p_{2}\right)
$$

Put $g=h_{1} g_{0}^{2} / 2, q_{1}=q_{0} p_{1}$ and $q_{2}=q_{0} p_{2}$, then $f=\left(q_{1}+q_{2}\right) g, g$ is strongly outer, $-i\left(q_{1}-q_{2}\right) /\left(q_{1}+q_{2}\right)$ is nonnegative and $\left(q_{1}-q_{2}\right)^{-1}$ is summable. By the lemma above $\operatorname{Im} \bar{q}_{1} q_{2} \leqq 0$.

If $q_{1}$ is a finite Blashke product of degree $n$ then so is $q_{2}$. For $-i\left(p_{1}-p_{2}\right) /\left(p_{1}+p_{2}\right)$ is a nonnegative in $N_{+}$because $p_{1}+p_{2}$ is outer, and

$$
\frac{-i\left(p_{1}-p_{2}\right)}{p_{1}+p_{2}}-i=\frac{-i 2 p_{1}}{p_{1}+p_{2}}
$$

and

$$
\frac{-i\left(p_{1}-p_{2}\right)}{p_{1}+p_{2}}+i=\frac{-i 2 p_{2}}{p_{1}+p_{2}}
$$

This shows degree $p_{1}=$ degree $p_{2}[6,7]$ and hence $q_{2}=q_{0} p_{2}$ is a finite Blaschke product of degree $n$.

In the theorem above

$$
-i\left(q_{1}-q_{2}\right) g=q_{0} q g_{0}^{2}
$$

In general even if $f=\left(q_{1}+q_{2}\right) g$ and $g$ is strongly outer, $-i\left(q_{1}-q_{2}\right)$ does not have such properties.

For any pair $\left(q_{1}, q_{2}\right)$ of inner functions $q_{1}, q_{2},-i\left(q_{1}-q_{2}\right) /\left(q_{1}+q_{2}\right)$ is real. However there exists a pair $\left(q_{1}, q_{2}\right)$ such that $-i\left(q_{1}-q_{2}\right) /\left(q_{1}+q_{2}\right)$ is nonnegative. Suppose $a, \alpha_{j}$ and $\beta_{j}$ are complex numbers, $0<|a| \leqq 1,\left|\alpha_{1}\right|<1$ and $\left|\alpha_{j}\right| \leqq\left|\beta_{j}\right|(j=1,2)$. If

$$
\alpha_{1} \beta_{1}=a / \bar{a}, \alpha_{1}+\beta_{1}=\left(1+|a|^{2}+i\right) / \bar{a}
$$

and

$$
\alpha_{2} \beta_{2}=a / \bar{a}, \alpha_{2}+\beta_{2}=\left(1+|a|^{2}-i\right) / \bar{a}
$$


then $-i\left(q_{1}-q_{2}\right) /\left(q_{1}+q_{2}\right)$ is nonnegative where

$$
q_{1}=\frac{z-\alpha_{1}}{1-\bar{\alpha}_{1} z} \quad \text { and } \quad q_{2}=\frac{z-\alpha_{2}}{1-\bar{\alpha}_{2} z}
$$

For $\quad|(z-a)(1-\bar{a} z) \pm i z|^{2}=|1-\bar{a} z|^{2}+1$. Hence $\quad(z-a)(1-\bar{a} z)+i z=q_{1} h \quad$ and $(z-a)(1-\bar{a} z)-i z=q_{2} h$ where $h$ is outer. Therefore $(z-a)(1-\bar{a} z)=\left(q_{1}+q_{2}\right) h$ and $z=-i\left(q_{1}-q_{2}\right) h$. Since $z /(z-a)(1-\bar{a} z)$ is nonnegative, $-i\left(q_{1}-q_{2}\right) /\left(q_{1}+q_{2}\right)$ is nonnegative.

\section{A sum of two inner functions and the square}

In this section we wish to factorize the part $q_{1}+q_{2}$ in Theorem 1 which is not strongly outer. This problem is suggested by a theorem of Helson [7] (see Section 1).

Theorem 2. Let $q_{1}$ and $q_{2}$ be inner functions such that $q_{1}+q_{2}$ is outer, $\operatorname{Im} \bar{q}_{1} q_{2} \leqq 0$ almost everywhere and $\left(q_{1}-q_{2}\right)^{-1}$ is summable. If the inner part $q$ of $q_{1}-q_{2}$ has one of the following three properties then there exist two inner functions $p_{1}, p_{2}$ such that

$$
q_{1}+q_{2}=\left(p_{1}+p_{2}\right)^{2} k
$$

where $k$ is strongly outer.

(1) $q=q_{0}^{2}$ for some inner function $q_{0}$.

(2) $q(U) \subsetneq U$.

(3) $q$ is a finite Blaschke product.

Proof. Since (1) is a special case of the proof of (2), we will show (2). If $q(U)_{\subsetneq} U$ then there exists $\alpha \in U$ with $\alpha \notin q(U)$. Put $q^{\prime}=(q-\alpha) /(1-\bar{\alpha} q)$ then $q^{\prime}=q_{0}^{2}$ for some inner function $q_{0}$ because $q^{\prime}$ is a singular inner function. Moreover

$$
\bar{q} q_{0}^{2}=\frac{\bar{h}_{0}}{h_{0}} \quad \text { and } \quad h_{0}=1-\bar{\alpha} q .
$$

Hence $\bar{q}\left(q_{0} h_{0}\right)^{2}=\left|q_{0} h_{0}\right|^{2}$ and $q_{0} h_{0} /(1+q)$ is real because $\bar{q}(1+q)^{2}$ is nonnegative. Let $s^{2}=q\left(q_{1}+q_{2}\right) /-i\left(q_{1}-q_{2}\right)$; then $s /(1+q)$ is real and $s \in H^{2}$ because $\left(q_{1}-q_{2}\right)^{-1}$ is summable. Since $\left|s \pm i q_{0} h_{0}\right|^{2}=|s|^{2}+\left|h_{0}\right|^{2}$,

$$
s+i q_{0} h_{0}=p_{1} h_{1} \text { and } s-i q_{0} h_{0}=p_{2} h_{1}
$$

where $p_{1}, p_{2}$ are inner functions and $h_{1}$ is a function in $H^{2}$ with $\left|h_{0}\right| \leqq\left|h_{1}\right|$. Hence $h_{1}^{-1} \in H^{\infty}$ and $s=\left(p_{1}+p_{2}\right) h_{1}$. Thus 


$$
q_{1}+q_{2}=\left(p_{1}+p_{2}\right)^{2} \frac{-i\left(q_{1}-q_{2}\right)}{q} h_{1}^{2}
$$

Put $k=-i\left(q_{1}-q_{2}\right) h_{1}^{2} / q$; then $k$ is strongly outer and hence (2) follows.

Suppose $q$ is a finite Blaschke product of degree $n$. If $f \in H^{1}$ and $\bar{q} f$ is nonnegative then $f=\gamma \prod_{j=1}^{n}\left(z-a_{j}\right)\left(1-\bar{a}_{j} z\right) l^{2}$ where $\gamma$ is positive constant, $\left|a_{j}\right| \leqq 1(1 \leqq j \leqq n)$ and $l^{2}$ is strongly outer in $H^{1}$ with $l^{-1} \in H^{\infty}([2,8])$. Since $s^{2}=q\left(q_{1}+q_{2}\right) /-i\left(q_{1}-q_{2}\right)$ is an outer function with $\bar{q} s^{2} \geqq 0$,

$$
s=\gamma_{1} \prod_{j=1}^{n}\left(-\bar{a}_{j}\right)^{1 / 2}\left(z-a_{j}\right) l
$$

where $\gamma_{1}>0$ and $\left|a_{j}\right|=1(1 \leqq j \leqq n)$. Put $f=\prod_{j=1}^{n}\left(-\bar{c}_{j}\right)^{1 / 2}\left(z-c_{j}\right) l$ where $\left|c_{j}\right|=1(1 \leqq j \leqq n)$ and $\left\{c_{j}\right\}_{j=1}^{n}$ is disjoint from $\left\{a_{j}\right\}_{j=1}^{n}$. Then

$$
|s \pm i f|^{2}=|s|^{2}+|f|^{2} \geqq \varepsilon|l|^{2}
$$

for some $\varepsilon>0$ because $\left\{c_{j}\right\}_{j=1} \cap\left\{a_{j}\right\}_{j=1}^{n}=\phi$. Therefore $s+i f=2 p_{1} h_{1}$ and $s-i f=2 p_{2} h_{1}$ where $p_{1}, p_{2}$ are inner functions and $h_{1}$ is a function in $H^{2}$ with $|l| \leqq\left|h_{1}\right|$. Now as in the proof of (2), (3) follows.

By Theorem 1 and the remark after it, if $f$ is outer and not strongly outer then

$$
f=\left(q_{1}+q_{2}\right) \frac{q}{-i\left(q_{1}-q_{2}\right)} g_{0}
$$

The proof of Theorem 2 implies that if $q$ satisfies one of (1)-(3) then $f$ can be factorized as in the following: $f=\left(p_{1}+p_{2}\right)^{2} h_{1}^{2} g_{0}$ and $h_{1}^{2} g_{0}$ is strongly outer. In Theorem 2 if $\bar{q} q_{0}^{2}=h_{0} / h_{0}$ and $h_{0}^{2}$ is strongly outer then the proof of (2) works and hence its conclusion is still valid.

\section{The solution sets of extremal problems}

For each $f$ in $H^{2}$ we define a subset of $H^{2}$ in the following:

$$
\mathscr{A}_{f}=\left\{g \in H^{2}: g f^{-1} \text { is real }\right\} \text {. }
$$

Again by virtue of Theorem 1 , we consider $\mathscr{A}_{q_{1}+q_{2}}$ for two inner functions $q_{1}, q_{2}$. If $\mathscr{A}_{q_{1}+q_{2}}$ contains a function whose absolute value is that of a strong outer function and the inner part satisfies the conditions in Theorem 2, then the conclusion of Theorem 2 is still valid. This is one of the motivations for the study of $\mathscr{A}_{q_{1}+q_{2}}$. For each $f$ in $H^{1}$ we define a subset of $H^{1}$ as follows: 


$$
\mathscr{S}_{f}=\left\{g \in H^{1}: g f^{-1} \text { is nonnegative }\right\} .
$$

The intersection of $\mathscr{S}_{f}$ and the unit ball $S^{1}$ of $H^{1}$ is a solution set of an extremal problem of $H^{1} . \mathscr{S}_{f} \cap S^{1}$ was described by the author [8] when it is weak-* compact and E. Hayashi $([4,5])$ described it completely. However the solution may not be understandable enough when it is not weak-* compact. If $f \in H^{2}$ then $\left(\mathscr{A}_{f}\right)^{2} \subset \mathscr{S}_{f^{2}}$ and this is another motivation for the study of $\mathscr{A}_{q_{1}+q_{2}}$.

Theorem 3. Let $q_{1}, q_{2}$ be inner functions and $p=q_{1} q_{2}$.

(1) $\mathscr{A}_{q_{1}+q_{2}}+i \mathscr{A}_{q_{1}+q_{2}}=H^{2} \ominus p z H^{2}$.

(2) Let $b$ be a Blaschke product such that $b=(p-\alpha) /(1-\bar{\alpha} p)$ and $\alpha \in U$ (such an $\alpha$ exists always). Then

$$
\mathscr{A}_{q_{1}+q_{2}}=(1-\bar{\alpha} p) \mathscr{A}_{1+b}
$$

(3) If $b$ is a finite Blaschke product of degree $n$ then

$$
\mathscr{A}_{1+b}=g^{-1} \mathscr{A}_{1+z n}
$$

where $g=\prod_{j=1}^{n} \bar{a}_{j}\left(1-\bar{a}_{j} z\right)$ and $\left\{a_{j}\right\}_{j=1}^{n}$ are the zeros of $b$.

(4) If $n$ is finite then

$\mathscr{A}_{1+z n}=\left\{\alpha \prod_{j=1}^{l}\left(z-a_{j}\right)\left(1-\bar{a}_{j} z\right) \prod_{j=2 l+1}^{n}\left(-\bar{a}_{j}\right)^{1 / 2}\left(z-a_{j}\right) ;\left|a_{j}\right|<1\right.$ if $1 \leqq j \leqq l$ and $\left|a_{j}\right|=1$ if $2 l+1 \leqq j \leqq n$, and $\alpha$ is real $\}$.

(5) $\mathscr{A}_{1+b} \supseteq \mathscr{A}=\left\{f \in H^{2}: f=\sum_{j=0}^{\infty}\left[\alpha_{j}\left(b_{j}+b_{j}^{\prime}\right)-i \beta_{j}\left(b_{j}-b_{j}^{\prime}\right)\right]\right\} \quad$ and $\quad \mathscr{A}+i \mathscr{A}=\mathscr{A}_{1+b}+$ $i \mathscr{A}_{1+b}$. Here $b=\prod_{j=1}^{\infty} b_{j}$ where

$$
b_{j}=\frac{\left|z_{j}\right|}{z_{j}} \frac{z-z_{j}}{1-\bar{z}_{j} z} \text { for } j \geqq 1,
$$

$b_{0}=1$ and $b_{j}^{\prime}=b \bar{b}_{j} . \alpha_{j}$ and $\beta_{j}$ are real numbers and $\sum$ denotes the $L^{2}$-limit of the finite sums.

Proof. (1) $\mathscr{A}_{q_{1}+q_{2}}=\mathscr{A}_{1+p}$ because

$$
\frac{q_{1}+q_{2}}{1+p}=\frac{\left(q_{1}+q_{2}\right) \bar{p}}{(1+p) \bar{p}}=\frac{\bar{q}_{1}+\bar{q}_{2}}{\bar{p}+1}
$$

If $f \in \mathscr{A}_{1+p}$ then $f(1+p)^{-1}=\widetilde{f}(1+\bar{p})^{-1}$ and hence

$$
(1+p)^{-1} H^{2} \cap(1+\bar{p})^{-1} \bar{H}^{2} \supseteq(1+p)^{-1}\left\{\mathscr{A}_{1+p}+i \mathscr{A}_{1+p}\right\} .
$$

If $(1+p)^{-1} f=(1+\bar{p})^{-1} \bar{g} \in(1+p)^{-1} H^{2} \cap(1+\bar{p})^{-1} \bar{H}^{2}$ then $p f=\bar{g}$ because $1+\bar{p}=\bar{p}(1+p)$. 
Hence $f=p_{1} h$ and $g=p_{2} h$ where $h$ is outer, and $p_{1}$ and $p_{2}$ are inner. This implies that $\bar{p} p_{1} h=\overline{p_{2} h}$ and $\bar{p} p_{2} h=\overline{p_{1} h}$. Therefore

$$
\bar{p}\left(p_{1}+p_{2}\right) h=\overline{\left(p_{1}+p_{2}\right) h}
$$

and

$$
\bar{p}\left\{-i\left(p_{1}-p_{2}\right) h\right\}=\overline{-i\left(p_{1}-p_{2}\right) h}
$$

This implies that $\left(p_{1}+p_{2}\right) h / 2$ and $-i\left(p_{i}-p_{2}\right) h / 2$ belong to $\mathscr{A}_{1+p}$. Since

$$
f=\left(p_{1}+p_{2}\right) h / 2+i\left\{-i\left(p_{1}-p_{2}\right) h / 2\right\},(1+p)^{-1} f(1+p)^{-1} \mathscr{A}_{1+p}+i(1+p)^{-1} \mathscr{A}_{1+p}
$$

Thus

$$
(1+p)^{-1} H^{2} \cap(1+\bar{p})^{-1} \bar{H}^{2}=(1+p)^{-1}\left\{\mathscr{A}_{1+p}+i \mathscr{A}_{1+p}\right\}
$$

On the other hand

$$
H^{2} \ominus p z H^{2}=H^{2} \cap p \bar{H}^{2}=(1+p)\left\{(1+p)^{-1} H^{2} \cap(1+\bar{p})^{-1} \bar{H}^{2}\right\}
$$

This shows (1) because $\mathscr{A}_{q_{1}+q_{2}}=\mathscr{A}_{1+p}$.

(2) If $l=1-\bar{\alpha} p$ then

$$
b=p \frac{|l|^{2}}{l^{2}}
$$

and hence $(1+p)^{2} l^{2} /(1+b)^{2}$ is nonnegative a.e.. Therefore $(1+p) l /(1+b)$ is real and $\mathscr{A}_{1+p}=\mathscr{A}_{(1+b) l}=l \mathscr{A}_{1+b}$ because $l^{-1} \in H^{\infty}$.

(3) $z^{n}=\bar{b}|g|^{2} / g^{2}$ and hence we can show $\mathscr{A}_{1+z^{n}}=g \mathscr{A}_{1+b}$ as in the proof of (2).

(4) Since $\left(\mathscr{A}_{1+z^{n}}\right)^{2} \subset \mathscr{S}_{z^{n}}$ and

$$
\mathscr{S}_{z^{n}}=\left\{\gamma \prod_{j=1}^{n}\left(z-a_{j}\right)\left(1-\bar{a}_{j} z\right):\left|a_{j}\right| \leqq 1(1 \leqq j \leqq n) \text { and } \gamma>0\right\}
$$

(4) follows.

(5) If $f=\sum_{j=0}^{\infty}\left[\alpha_{j}\left(b_{j}+b_{j}^{\prime}\right)-i \beta_{j}\left(b_{j}-b_{j}^{\prime}\right)\right]$ then it belongs to $\mathscr{A}_{1+b}$ by the first line of (1) and

$$
\frac{-i\left(b_{j}-b_{j}^{\prime}\right)}{1+b}=\frac{-i\left(b_{j}-b_{j}^{\prime}\right) \bar{b}}{(1+b) \bar{b}}=\frac{i\left(\bar{b}_{j}-\bar{b}_{j}^{\prime}\right)}{\bar{b}+1}
$$

If $f \in H^{2} \Theta b z H^{2}$ and $f$ is orthogonal to $\left\{b_{j}+b_{j}^{\prime},-i\left(b_{j}-b_{j}^{\prime}\right)\right\}_{j=0}^{\infty}$ then it is orthogonal to $\left\{b_{j}, b_{j}^{\prime}\right\}_{j=0}^{\infty}$. We will show that $f$ is zero almost everywhere. Since $\left(f, b_{0}\right)=0$ and $b_{0}=1$, 
$f=z f_{0}$ and $f_{0} \in H^{2} \ominus b z H^{2} .\left(f, b_{1}\right)=0$ implies $f_{0}\left(z_{1}\right)=0$, and hence $f_{0}=b_{1} f_{1}$ and $f_{1} \in H^{2}$, where (,) denotes the inner product in $H^{2}$. Since

$$
0=\left(f_{0}, b_{2}\right)=\left(f_{1}, b_{2} \bar{b}_{1}\right)
$$

$f_{1}\left(z_{2}\right)=0$ and $f_{0}\left(z_{2}\right)=0$. By the same argument, $f_{0}\left(z_{j}\right)=0$ for $j \geqq 3$ and hence $f \in b z H^{2}$. This implies that $f$ is zero because $f \in H^{2} \ominus b z H^{2}$. This completes the proof of (5).

\section{A characterization of strong outer functions}

Several characterizations of strong outer functions are known (cf. [8, Theorem 3], [4, Theorem 8], [3, Chapter IV, Exercise 18] and [7]). See Theorem 1. In this section we give a characterization. For a real valued measurable function $u$ on $\partial \mathrm{U}$, if $f \in N$ and $\operatorname{Re} f=u$ on $\partial U, \tilde{u}$ denotes the real part of $f$ on $\bar{U}$, that is, the harmonic extension of $u$, and * $u$ denotes the imaginary part of $f-f^{\prime}(0)$ on $\bar{U}$.

If $f$ is in $H^{1}$ and $u$ is a nonnegative function in $L^{1}$ such that $\left|f\left(e^{i \theta}\right)\right| \leqq u\left(e^{i \theta}\right)$ almost everywhere on $\partial U$, then $|f(z)| \leqq \tilde{u}(z)$ on $U$. The inner outer factorization of $f$ and the Poisson integrals of $u$ and $|f|$ give the above inequality in $U$. If $u$ is nonnegative then $u+i^{*} u$ belongs to $\bigcap_{p<1} H^{p}$ and is an outer function. Let $f\left(e^{i \theta}\right)=\left(e^{i \theta}-1\right)^{2}$ and $u\left(e^{i \theta}\right)=-2\left(e^{i \theta}-1\right)^{2}\left(e^{i \theta}+1\right)^{-2}(1+\cos \theta)$; then $u+i^{*} u=-2\left(e^{i \theta}-1\right)^{2}\left(e^{i \theta}+1\right)^{-1}$ is an outer function in $\bigcap_{p<1} H^{p}$ and $\left|f\left(e^{i \theta}\right)\right| \leqq u\left(e^{i \theta}\right)$ a.e. on $\partial U$. However it is not true that $|\tilde{f}(z)| \leqq \tilde{u}(z)$ on $U$. Hence the condition of the integrability of $u$ is needed to show the inequality on $U$.

Theorem 6. Let $g$ be an outer function in $H^{1}$. The following are equivalent.

(1) $g$ is a strong outer function.

(2) For every nonnegative function $u$ on $\partial U$ such that $u+i^{*} u$ is an outer function in $N_{+}$ and $\left|g\left(e^{i \theta}\right)\right| \leqq u\left(e^{i \theta}\right)$ a.e. on $\partial U$, we have $|\tilde{g}(z)| \leqq \tilde{u}(z)$ on $U$.

(3) For every nonnegative function $u$ on $\partial U$ such that $u+i^{*} u$ is an outer function in $N_{+}$ and $\left|g\left(e^{i \theta}\right)\right| \leqq u\left(e^{i \theta}\right)$ a.e. on $\partial U$, we have $|\tilde{g}(0)| \leqq \tilde{u}(0)$.

Proof. The proof is suggested by the proof of [3, Chapter IV, Lemma 5.4].

$(1) \Rightarrow(2)$. Put $\phi=u+i^{*} u$. Let $\alpha$ be a measurable function on $\partial U$ such that

$$
|\alpha| \leqq \pi / 2 \text { a.e. } \quad \text { and } \quad \alpha=\arg \phi(\bmod 2 \pi)
$$

Then $u=\operatorname{Re} \phi=|\phi| \cos \alpha$ and $|g / \phi| \leqq \cos \alpha$ a.e. on $\partial U$. Let $\psi=\exp \left(-{ }^{*} \alpha+i \alpha\right)$ then $\psi$ belongs to $H^{p}$ for some $p<1$ by Zygmund's theorem (cf. [3, Chapter III, Corollary 2.6]) and

$$
|\psi g / \phi| \leqq|\psi| \cos \alpha=\operatorname{Re} \psi \text { a.e. on } \partial U
$$


and $\operatorname{Re} \psi$ is in $L^{1}$. Since $\phi$ is an outer function, $\psi g / \phi$ is in $N_{+}$and hence $\psi g / \phi$ belongs to $H^{1}$. Put $h=\psi g / \phi$. Then $\arg h=\arg g$ a.e. $(\bmod 2 \pi)$ and hence $h=\gamma g$ for some $\gamma>0$ because $g$ is strongly outer. Thus $\psi=\gamma \phi$ and $u$ is in $L^{1}$. By the remark above Theorem 6 $|\tilde{g}(z)| \leqq \tilde{u}(z)$ on $U$. This implies (2).

$(2) \Rightarrow(3)$ is trivial.

$(3) \Rightarrow(1)$. If $g$ is not a strong outer function, then there exists an outer function $h_{0}$ in $H^{1}$ such that $\arg g=\arg h_{a, \gamma}$ a.e. $(\bmod 2 \pi)$ for any $|a|=1$ and $\gamma>0$ where $h_{a, \gamma}=$ $\gamma(z-a)(1-\bar{a} z) h_{0}$. We can choose $a$ and $\gamma$ such that

$$
\operatorname{Re} h_{a, \gamma}^{-1}(0) \tilde{g}(0)<-|\tilde{g}|(0) .
$$

Hence, if $u=|g|+\operatorname{Re} h_{a, y}^{-1} g=h_{a, y}^{-1} g(|g|+1)$ then

$$
\tilde{u}(0)=|\tilde{g}|(0)+\operatorname{Re} \tilde{h}_{a, \gamma}^{-1}(0) \tilde{g}(0)<0 \leqq|\tilde{g}(0)| .
$$

On the other hand,

$$
h_{a, \gamma}^{-1} g\left(\left|h_{a, \gamma}\right|+1\right)+i h_{a, \gamma}^{-1} g^{*}\left(\left|h_{a, \gamma}\right|+1\right)
$$

is analytic and outer in $N_{+}$. This contradicts (3).

\section{REFERENCES}

1. A. Buerling, On two problems concerning linear transformations in Hilbert space, Acta Math. 81 (1949), 239-255.

2. K. DE Leeuw and W. Rudin, Extreme points and extreme problems in $H^{1}$, Pacific J. Math. 8 (1958), 467-485.

3. J. Garnett, Bounded Analytic Functions (Academic Press, New York, N.Y., 1981).

4. E. Hayashi, The solution sets of extremal problems in $H^{1}$, Proc. Amer. Math. Soc. 93 (1985), 690-696.

5. E. Hayashi, The kernel of a Toeplitz operator, Integral $E_{q}$. Operator Th. 9 (1986), 589-591.

6. H. Helson, Large analytic functions, Oper. Theory: Adv. Appl. 43 (1989), 209-216.

7. H. Helson, Large analytic functions II, in Analysis and Partial Differential Equations (C. Sadosky, ed., Marcel Dekker, Basel, 1990).

8. T. NAKAZI, Exposed points and extremal problems in $H^{1}$, J. Funct. Anal. 53 (1983), 224-230.

Department of Mathematics

Faculty of SCIENCE

HOKKaIDO UnIVERSITY

SAPPORO 060

JAPAN 